\title{
UNA IMPOSTAZIONE DELL'OPERA DI ELSA MORANTE
}

\author{
Carolina Massi Albanese \\ Universidade Federal do Paraná
}

\section{RIASSUNTO}

Il presente studio abbraccia tutta l'opera di Elsa Morante includendo il romanzo Aracceli pubblicato da pochi -mesi.

Sono messi in rilievo i nuclei caratterizzanti dell'opera morantiana: la collocazione dell'uomo in una dimensione condizionante, la sensazione del mistero della vita e del nostro inutile interrogarla, il conseguente disorientamento angoscioso dell'uomo e la dilatazione fiabesca.

Nelle prime opere la narrativa si produce in una sperequazione tra stupefatto lirismo consonante con un'atmo. sfera surreale, ma questa reinvenzione della realtà scaturisce da una lucida lettura "a rovescio" della realtà le cui immagini sono voluntamente distorte. A partire da II mondo salvato dai ragazzini, le illusioni e le visioni de L'isola di Arturo, così come la "menzogna" e il "sortilegio" del romanzo omonimo, sono sostituiti dalla rivelazione impietosa del mondo: è smantellato il "grande carnevale" della storia. Tuttavia, se nel penultimo romanzo: La Storia, gli anticon. formisti e gli umili, quasi relitti di naufragio, ci insegnano a riconoscere la verità e la bontà della natura, nella recente produzione: Aracoeli non si ammette più nulla. Il romanzo si riduce ad essere un monologo sregolato.

Elsa Morante ha sempre custodito gelosamente la propria vita privata. Restia agli avvenimenti mondani, "selvatica, astorica e anarchica",' l'insoddisfazione del quotidiano è riempito e trasfigurato ancor oggi da letture e viaggi.

La scrittrice nacque a Roma nel 1918, da padre siciliano e madre dell'alta Italia. Il padre era institutore in un riformatorio, mentre la madre faceva la maestra elementare. Il

1 MANACORDA, O. Morante. In: —_- Storia della letteratura italiana contemporanta. Roma, Ed, Riunitl, 1972. p. 329. 
suo "curriculum" scolastico fu alquanto irregolare: la Morante fu sostanzialmente autodidatta. Trascorse l'infanzia nel quartiere popolare del Testaccio che trent'anni dopo sarebbe diventato uno dei poli del mondo poetico del Pasolini. Nel 1941 sposò Alberto Moravia. Moravia, nonché l'amicizia con Umberto Saba, Sandro Penna e Pier Paolo Pasolini, sono stati significativi per la Morante. Nel 1943 la guerra la costrinse a vivere per circa un anno tra i contadini ed i fuggia. schi del fronte di Cassino. In questi luoghi avvenne il primo contatto diretto della scrittrice con il Sud. Si tratta di un avvenimento importante perché l'interesse per la mentalità del Mezzogiorno è una delle caratteristiche salienti della sua narrativa.

La precocità narrativa di Elsa Morante è testimoniata da Le straordinarie avventure di Caterina fiabe scritte all'età di tredici anni. Si parla di un palazzo di sogni dove tutti i bambini, anche gli straccioni, possiedono un sontuoso appartamento. Qui la Morante tredicenne utilizza tecniche narrative che porteranno in seguito a maturazione certe scelte stilistiche, ora appena individuabili, ossia: l'aggettivazione qualificante e impositiva di un giudizio; il lirismo trasognante tendente allo splendido e al meraviglioso.

Tit non ha una casa, ma foreste e praterie con tigri e alberti del pane e uccelli del Paradiso dalle ricche piume. Palmizi lunghi e sottili sorgono a grandi distanze per il deserto, e da un lato si leva una montagna oscura in cui si nascondono i banditi fra un precipitare di torrenti. In un chiostro che odora di aranci e digiacinti, presso fontane bianche passeggiano molte principesse coi capelli chiusi in reti d'oro.'

La congiuzione "e" del primo periodo genera un movimiento delicatamente ondoleggiante, mentre i sostantivi invocano mondi favolosi e meravigliosi e l'aggettivazione è preziosa.

Tit è uno straccione coraggioso come un principe ed uccide belve e affronta navi corsare, ed anche gli altri personaggi rievocano atmosfere fiabesche, oniriche, visionarie.

Da questa informe preistoria della narrativa morantiana, si arriva alla narrativa vera e propria con i racconti de Il gioco segreto. ${ }^{3} \mathrm{Vi}$ si trovano atmosfere oniriche, la tendenza all'evasione fantastica ed alla trasformazione teatrale della

2 MORANTE. Elsa. Le straordinario avventure di Caterina. Torino, Einaudi, 1941. p. 4.

3 MORANTE. Elsa. Il gtoco segreto. Milano. Garzantl, 1941. $422 \mathrm{p}$. Le citazionl al riferiscono a questa cdizione e saranno citati solo 1 numerl delle paglne. 
realtà. É la metaforizzazione della vita nel teatro, l'evasione dal reale attraverso la fantasia e la recitazione.

Esaminiamo il primo racconto, Il ladro dei lumi, scritto nel 1935. Dalle finestre del quinto piano una bambina si mette ad osservare ciò che accade nell'interno di un tempio ebraico posto dinanzi alla sua abitazione. Ella scopre cosi che Jusvin, il guardiano, ruba l'olio delle lampade: "Dalla finestra potevo scorgere il Tempio, la sua cupola tozza, le lunghe finestre dai vetri colorati, e attraverso i vetri, l'opaco rosseggiare delle lucerne dei morti" (p. 2). Da osservare la significazione del cromatismo aggettivale per definire lo spazio e riempirlo di tonalità antagonistiche.

La bimba, spaventata, è sicura che Dio punirà il guardiano del tempio: "Iddio lo vedeva e avrebbe pensato a punire colui che rubava la luce dei morti. Iddio farà giustizia" (p. 5). Per lei Dio è sempre una presenza minacciosa e vendicativa e se l'uomo pecca, la punizione non è rinviabile:

Il dito del Signore lo aveva toccato sulla lingua, ed ora quella lingua maledetta di Jusvin si disfaceva in una piaga. Ecco Jusvin in cospetto del Signore. Il Signore non ha né corpo né faccia; è come una nube di tempesta, come la ombra di una montagna: Pietà, Signore, l'ho fatto per i miei figli. Acqua alla mia lingua, sonno ai miei occhi. Pietà del mio camminare che invidia i placidi morti. Parole sono queste che ha sepolte nella gola, ma non prenderanno mai form asulle sue labbra. La bocca si torce, gorgoglia, l'uomo gestisce e suda. E lui il senzaforma non parla. Il suo tacere significa: Tu, ladro (p. 10).

Anche la bambina si sente colpevole per aver perduto il biglietto con i numeri giocati al lotto: "Io giravo guardando le case, le finestre e le facce, senza pensare al biblietto, e ho peccato. Anche Jusvin aveva peccato e il Signore l'ha punito" (p. 14).

La condizione qui è analoga alla precedente: al peccato segue la punizione: "Così Dio, nel mezzo, il silenzioso, che castiga i vivi e rinchiude nella terra i morti" (p. 15). E la bimba, posseduta da un forte complesso di colpa, pensa di fuggire, di andare via dalla sua dimora, dalla sua città, e di morire:

Tale era il mio Dio; e quella ragazzina fui io, o forse mia madre, o forse la madre di mia madre; io sono morta e rinata, e ad ogni nascita si inizia un nuovo processo incerto. E quella ragazzina è sempre là, che interroga spaurita nel suo mondo incomprensibile, sotto l'ombra del giudice fra i muti" (p. 16). 
Questo racconto rivela nuclei caratterizzanti dell'opera morantiana: la collocazione dell'uomo in una dimensione condizionante, la sensazione del mistero della vita e del nostro inutile interrogarla, il conseguente disorientamento angoscioso dell'uomo e la dilatazione fiabesca.

Soffermiamoci ora sul racconto Il gioco segreto che dà il titolo alla raccolta. Il racconto inizia con la descrizione di un palazzo abitato dai Marchesi, nobilucci rovinati economicamente ma ostinati nella difesa dei privilegi di casta. Nel palazzo in rovina tre giovani eredi, Antonietta, Giovanni e Pietro evadono dal reale attraverso il gioco del teatro. Essi attendono sempre la notte per poter recitare, per poter scatenare il bisogno di una compensazione alla realtà quotidiana mediocre e sgraziata. I ragazzi si identificano con i personag. gi dei libri di avventura e con quelli dipinti sulle decrepiti pareti del palazzo:

Nell'interno del palazzo si seguivano grandi sale vuote in cui nei ventosi giorni di tempesta, entra. vano dai vetri rotti mulinando la polvere e la pioggia. Dalle pareti pendevano lembi strappati di tappezzerie, avanzi di arazzi logori, e nei soffitti, fra le nuvole gonfie e smaglianti, navigavano cigni e angioli nudi, e donne splendide si affacciavano entro ghirlande di fiori e di frutti. Alcune sale erano affre. scate di avventure e di storie, e vi abitavano popoli regali, che montavano cammelli o giocavano in folti giardini fra scimmie e falchi (p. 78).

"Fu così che i tre fanciulli scoprirono il teatro. I loro personaggi uscirono del tutto dalla nebbia dell'invenzione, con suono d'armi e frescio di vesti. Acquistarono un corpo di carne ed una voce, e per i fanciulli cominciò una doppia vita (p. 83).

Ecco quindi che all'iniziale fantasticare subentra l'identificazione dei personaggi dei libri con quelli dipinti sulla parete e finalmente l'immedesimazione dei ragazzi con i personaggi:

Antonietta era in piedi presso un albero dipinto nel quale d'improvviso cominciò a correre la linfa. Uccelli addormentati ma vivi giacquero tra le foglie. $E$ su lei per miracolo crebbe una veste lunga, di forma sontuosa e vegetale, da cui pendeva una borsa d'oro. I suoi capelli si divisero in due trecce bionde, e le sue pupille si dilatarono per il rapimento e per la paura (p. 87). 
Deliranti, eterni sonnambuli, i tre nella recitazione evocano l'universo fantastico; ecco però che la recita è bruscamente interrotta dai genitori che sgridano i ragazzi per le loro stolte fantasticherie. Mentre Antonietta e Pietro sopportano il rientro alla normalità, Giovanni, il più giovane, un corpo misero e avvizzito con strane febbri epilettoidi, continua nella sua fuga dal reale trascinandosi nell'incatesimo notturno, in una meta chimerica "Arriverò a quella catena di monti-egli pensò e poi al mare" (p. 92).

Il racconto ha molti punti di contatto con Menzogna e Sortilegio: il tema dell'evasione dal mondo. La Morante fornisce i mezzi per realizzarla: fantasia, finzione, ciò nonstante avverte che la favola si trasformerà inesorabilmente in menzogna e illusione, se non sarà accompagnata dalla coscienza. Ecco perché i tre ragazzi, a differenza di Elisa (la protagonista di Menzogna e Sortilegio), nell'abbandono inconsapevole saranno bruciati dalle stesse luci del teatro.

Menzogna e Sortilegio ${ }^{4}$ è il primo romanzo di Elsa Morante. Pubblicato nel 1948 vince il premio Viareggio. L'azione del romanzo si svolge in un campo sentimentale e storico cupamente siciliano caratterizzato dalla superstizione e dalla megalomania. Tuttavia, i personaggi oltre ad esporre la mentalità dell'Italia del Sud, sono soprattutto emblemi dell'incapacità degli uomini di sopportare la propria condizione terrestre e della necessità di evadere verso miti e superstizioni.

La narrativa si produce in una sperequazione tra stupefatto lirismo consonante con un'atmosfera surreale. Il critico Falqui notava che in questo libro "il linguaggio non serve né tende a rivelare la realtà, bensì mira a suscitare un tempo di sogno"."

Elisa, la protagonista, una ragazzina siciliana orfana, è portata a Roma da Rosaria, una prostituta generosa, ex amante del padre di Elisa. La ragazzina passa i giorni tra letture fantastiche "in cui l'esistenza terrestre non è descritta quale si mostra ogni giorno ai mortali assennati; bensì piena di prodigi, di stravaganza e di follia (p. 18). Elisa fa dei suoi parenti morti, fantasmi che la incitano a stravolgere la realtà nella finzione. "Dopo essersi fatte creder la mia consolazione, la mia festa e il mio riscatto di contro alla inquietante realtà, le mie maschere m'imposero la negazione dogni realtà, in cambio del loro mondo larvale" (p. 25).

Morta Rosaria, Elisa ormai sola al mondo, è abbandonata anche dai falsi eroi defunti: le loro magnanime imprese

4 MORANTE, Elsa. Menzogna e sortlleglo. Torino, Einaudl, 1948. 621 ๖. Le citazlonl si riferiscono a questa ediztone e saranno citatl solo 1 numerl delle paglne.

5 FALQUI, E. Morante. In: __ . I Contemporanei. Mulano, Marzoratl.

5 FALQUI, E. Morante. In: - Novecento letterario. Firenze, Vallecchl, 1961. p. 221. 
e grandi gesta si rivelano vicende di miseri personaggi. "E se li cerco, io credo di scorgere per la camera sconvolta in luogo delle loro abbaglianti figure, flosce spoglie senza vita. Simili ai costumi abbandonati in disordine dagli attori, alla fine dello spettacolo, in un retroscena d'infimo teatro (p. 32).

Elisa indaga ora la vita dei suoi parenti e soprattutto perché essi si sono fatti adoratori della menzogna, perché si sono fatti distruggere dalle loro stesse ambizioni, rifiutando il loro reale destino, convertendo la menzogna in sortilegio.

Il teatro è il limite della demarcazione tra verità e menzogna: simbolo dell'irrealtà e specchio di una realtà che è menzogna. Ma anche Elisa, come i parenti fantasmi, vive di menzogna e di sortilegio e se non si autodistrugge con megalomanie sociali come i primi, si segrega dal mondo per ricostruire, trasfigurandole, le vicende dei suoi familiari.

Le due funzioni di Elisa personaggio e narratrice si riflettono nei due piani temporali della vicenda: la dimensione cronologica tradizionale e la dimensione di un tempo puramente interiore che scaturisce dall'intervento della veggenza e del ricordo di Elisa sui fatti. La compresenza dei due piani narrativi dà al romanzo un impianto memoralistico appena esteriormente: il passato ed in certo modo anche il futuro acquistano realtà solo nel presente:

Il passato e il futuro, infatti, sono due i campi di nebbia e di vertigine, che i vivi non possono esplorare se non con la fantasia e con la memoria; ma forse fantasia e memoria sono soltanto strumenti d'illusione, e soltanto per un giuoco ingannevole l'uomo crede di avere il passato alle spalle e il futuro innanzi a sé. In realtà, egli si muove sopra una sfera immobile, conchiusa fin da principio, e il pas. sato e il futuro sono tutt'uno. A che serve esplorare questa reggia della morte? Il solo tentativo di sondarla produce angoscia e nausea, come quando ci si affaccia su un precipizio" (p. 233).

Elisa è allo stesso tempo io narrante e io recitante: narratrice-spettatrice è anche personaggio e anzi, protagonista dell'azione. Elisa ricostruisce la vicenda e narra i fatti seguendo una linea cronologica di sviluppo segnata dalle operazioni associative che compie la coscienza. Ecco quindi che il tempo non ha importanza in sé, osserviamo i rimandi del narratore, le parentesi sospensive, interventi della veggenza e del ricordo di Elisa sui fatti. Parte della vicenda è ricomposta sulla base di testimonianze o addirittura di documenti, per esempio: il carteggio Anna-Edoardo, il primo socialismo, 
il lume a petrolio e poi la luce elettrica, il dirigibile, il vagone postale. Notiamo anche le precisazioni dei tempi di ogni avvenimento: della morte di Teodoro, dell'incontro di Anna con Edoardo, della vedovanza di Damiano e così via. Eppure, i momenti così meticolosamente definiti diventano campi di nebbia: gli spostamenti dal tempo presente al passato remoto, dal tempo imperfetto al passato prossimo, genera una confusione di tempi per cui si deduce che cio che importa non è il fluire metodico del tempo ma sì il senso del destino: il tempo reale è il tempo interiore.

Come il tempo, anche lo spazio costituisce un altro elemento portante della narrazione. Tutti gli spazi sono limitati, definiti; la camera della nonna "cosi stretta che oltre al letto non c'era posto per un armadio" ( p. 42); e il palazzo Ceretano: "Il vestibolo, in penombra, scintillante di specchi e di marmi screziati, si termina a destra con questa scala" (p. 659); la casa di Rosaria: stanze "semibuie a causa delle scarse finestre e dei tendaggi" (p. 553); la casa coniugale di Anna; "tre camere ripiene, ma in una misura all'incirca tre metri nel suo lato più lungo e la cucina è un rettangolo piuttosto buio, dai muri affumicati" (p. 507).

Lo spazio è anche etnico: una canzone popolare, una espressione dialettale. Ma questo spazio tende sempre a delinearsi come spazio metaforico perché riflette la condizione storica ed esistenziale; la casa nuziale di Anna, per esempio: "Sul fondo di questo terreno disordinato, sterile, diviso da recinti di ferro spinato, si levava una brutta montagna tutta sparsa di cocci e di vetro" (p. 429).

Ferro spinato, carcere, carcere-prigionia, sono parole che si rincorrono lungo il romanzo: casa-carcere e spazio chiuso in cui il personaggio s'illude di muoversi. Dirà il personaggio Edoardo: "In realtà egli (l'uomo) si muove sopra una sfera immobile, conchiusa fin da principio" (p. 233). Questa impossibilità di muoversi e di comunicarsi è quindi impossibilità di relazione e d svolgimento: un'esistenza assurda. Si vedano alcune espressioni: "Si accorse di trovarsi in un vuoto spietato e senza rimedio" (p. 33). "I giorni erano del resto tanto eguali da potersi confondere l'uno con l'altro" (p. 49).

"La vita mortale, rinchiusa fra il tempo e lo spazio, è una prigione per loro, che incessantemente si agitano fra queste angustie, illusi forse di ripercorrere i liberi campi originari" (p. 164).

Alcune rare volte lo spazio si allarga ma tende sempre alla desolazione:

Distese di campi senza confine visibile di acque né di montagne. Non aranceti, né palme, né pinete, 
rocce marine, nessuna di taliglorie del Mezzogiorno dava pregio a questi campi. Monotoni e disadorni, essi alternavano i bruni maggesi ai prati, magri per la scarsità d'acqua. Di rado un orto o un vigneto spoglio interrompeva la pigra landa" (p. 77).

Ed anche l'aggettivazione rincara la dose dell'abbandono: "Le strade erano ampie e semivuote e dalle strette finestre in alto entravano luci livide e sghembe" (p. 386).

Tutti i personaggi di Menzogna e Sortilegio per rappresentare il proprio dramma hanno bisogno del teatro: Concetta, che nella superbia della sua umiltà, trasforma la chiesa in un "divino" teatro; Anna, che nella recita delle lettere, si crea un "teatro impercettibile, divino, ove si rappresentava per lei sola un'opera commovente e deliziosa", Elisa, che assiste come ad una "rappresentazione di gala" alle finzioni di Anna e Concetta. Teatro è pure il litigio tra Francesco e la moglie: l'una che si accusa di un falso adulterio e l'altro che nella sua gelosia da Otello "tiene teatro anche di notte. E Anna mo rirà tra i suoi "chimerici teatri" Ma il più sottile, beffardo regista di drammi, rimane Edoardo che organizza la suprema rappresentazione della menzogna in un diabolico teatro dove con la sua assen$\mathrm{za}$, con la sua morte, diventa il "deus ex machina "di tutta la vicenda.

La metafora del teatro è centrale nell'economia del romanzo; addirittura il teatro, specchio della condizione umana, "maximum" della finzione, luogo deputato della menzogna."

Menzogna e Sortilegio va interpretato come un'opera sulla crisi spirituale dell'uomo e sulla frattura tra io e mondo. La menzogna è diventata l'ideologia di una società malata che si nutre di miti per non aver più valori in cui credere e per i quali agire.

Il primo romanzo morantiano Menzogna e Sortilegio è stato giudicato da Lukács il massimo romanzo italiano moderno e citato dallo stesso fra i migliori esempi narrativi apparsi in Occidente dopo la Rivoluzione d'Ottobre. Per Lukács la Morante esprime una rivolta, ancora solitaria e individuale, alle forme di alienazione e alla sclerosi sociale pro- 
vocata dal crollo dei valori su cui poggiava l'organizzazione borghese della società.?

Il suo secondo romanzo: L'isola di Arturo, ha ottenuto maggior fama. L'isola di Arturo è stato tradotto in molte lingue, come del resto tutte le opere morantiane. Pubblicato in 1957 riceve il premio Strega. Vi si parla del paradiso dei bambini, del mondo non alienato da preconcetti sociali. Arturo vive a contatto con la natura seguendo liberamente le proprie fantasie eroiche fino a raggiungere maturità e pertanto la fine del'età felice.

Arturo, orfano di madre, trascorre la sua fanciullezza nell'isola di Procida, affidato al balio Silvestro ed al rozzo domestico Constante visto che il padre, Wilhelm, è quasi sempre assente. In assoluta libertà il ragazzo si sente re nella sua isola e riversa la carica emotiva degli affetti sul genitore la cui figura è rivestita di un carattere mitico. Wilhelm un giorno conduce nell'isola la sua nuova moglie, Nunziatina, una popolana sedicenne di Napoli. Le avvenute nozze sono per Arturo una terribile rivelazione, $\mathrm{ma}$ il disprezzo che egli sente per la giovanissima matrigna va via via trasformandosi in amore incestuoso. Arturo finisce col dichiarare apertamente il suo amore ma Nunziatina lo respinge con orrore. A questa dolorosa prova segue un'altra ancor più dolorosa, il ragazzo scopre che il padre è un omosessuale. É la fine dell' adolescenza di Arturo il quale, abbandonata l'isola, vi ritornerà tuttavia con la mente e con il cuore.

Questo romanzo dà l'impressione di continuare la tematica incontrata in Menzogna e Sortilegio perché ambientato come il primo nell'Italia meridionale, è anche caratterizzato dalla mescolanza di realtà e di favola, dalla mitizzazione delle persone amate, dal disperato ricorso all'illusione. C'è, però, una differenza molto significativa: mentre nel primo romanzo Elisa cancella con la menzogna le miserie della vita, ma diventa schiava dei suoi stessi sortilegi, Arturo segue liberamente le proprie fantasie vivendo una vita intatta e felice, al di là di ogni conoscenza e distinzione di bene e di male, fino al risvolto doloroso che gli fa raggiungere la maturità.

Nel secondo romanzo la Morante rafforza la sua concezione della vita come di un sogno e di un mistero. Al diventare adulto, l'essere umano assiste al disfacimento dei propri sogni ma non del mistero, quest'ultimo infatti rimarrà ancor più denso e terribilmente angoscioso perché svanisce la speranza di svelarlo.

Le prime settanta pagine del libro parlano della vita di Arturo fino al quattordicesimo anno di età, ossia, l'antece- 
dente della vicenda; le altre trecento venti rivestono due anni di vita, fino al sedicesimo. Gli otto capitoli, didascalicamente enunciati, constituiscono i momenti segnaletici dell'andatura del romanzo.

Già dalla dedica del romanzo si capta la tesi da dimostrare: "Quella che tu credevi un piccolo punto della terra, fu tutto. $E$ tu non saprai la legge ch'io, come tanti, imparo, e a me ha spezzato il cuore: fuori del limbo non v'è eliso"."

Anche il verso di Saba, all'inizio del volume, è programmatico: "Io, se in lui mi ricordo, ben mi pare..."

Il verso è tratto da Il fanciullo appassionato, e continua così:

che può fargli più niente altro vedere che il suo mondo, anche in vista impallidire come un appassionato, dargli un bello diverso che di un giovane animale?

Io, se in lui mi ricordo, ben mi pare che il suo cuore non debba ancora sapere quella che in ogni nostra cura è ascosa, malinconia amorosa.

Meglio in un lungo avventuroso sogno il suo ben corrucciato occhio s'interna. Anche gli è noia la casa paterna, Un carcere la scuola; e forse è nulla di tutto questo; è appena un6ombra vana che insegue, un indistinto ancor bisogno di esplorare più addentro che la bbrulla collina, e il porto, e lunghe vie remote.?

E soprattutto l'isola "Ia metafora geografica" 10 della mitica infanzia di Arturo. L'isola ha un aspetto primitivo, il mare che la circonda sembra far da barriera alla storia ed al tempo. Persino il nome del ragazzo è mitizzato: Arturo è il nome del mitico re dei cavalieri della tavola rotonda. La propria Morante in Risposta precisa che ha affidato al nome Arturo un "valore araldico" emblematico dell'intera umanità. Arturo è uno di questi eroi-semidei che riscatta per l'umanità minacciata la plaga felice dell'infanzia e dell'adolescenza del mondo. ${ }^{11}$

Numerosi sono gli archetipi mitici, particolarmente cari alla Morante. Orfano di madre, Arturo è allevato dal balio Silvestro con latte di capra. Quando arrivano nell'isola i pa-

8 MORANTE, Elsa. L'isola di Arturo. Torino, Elnaudi, 1937. p. 2.

9 SABA. U. Il fanciullo appassionsto. In: - "Canzoniere. Torino, Elnaud!, 1961. p. 321

10 PUPINO. p. 723

11 MORANTE, Elsa. Risposts a dieci voci per 11 bllenzlo. Europa Lotteraria, Roma, 30 gen. 1964. 
renti materni, il balio nasconde il bambino in una cassetta con il succhietto ed il latte, affinché non pianga allo svegliarsi e non sia scoperto dai parenti che potrebbero portarlo via.

Allorché Nunziatina lo respinge, Arturo fugge e si nasconde in una grotta sulla riva del mare; arriva l'ex-balio e quale inviato degli dei lo aiuta a separarsi dall'isola.

Anche qui chi narra è un personaggio: Arturo uomo, che si intrattiene unicamente sulla sua infanzia ed adolescente senza mai parlare di sé como adulto:

A distanza di tanto tempo, adesso io vado tentando di capire i sentimenti che, in questi giorni, cominciavano ad accavallarsi stranamente nel mio cuore; ma tuttora mi trovo incapace di distinguere le loro forme, che si mischiavano in disordine dentro di me, e non erano illuminate da nessun pensiero. Al ricordo mi sembrava di scorgere una valle, una turba di lupetti, o leoni.

e più avanti dirà:

(Si badi bene: non era che lo considerassi un dono troppo modesto, o ridicolo per la sua poca importanza! No, a quel tempo io avevo sui valori delle cose, delle idee strane, che non rispondevano alla realtà) ."

Il frequente uso dell'interrogativa e la virgolazione sono spie della disposizione meditativa di Arturo sui fatti della sua infanzia ed adolescenza.

Arturo uomo, diversamente da Elisa in Menzogna e Sortilegio, non cerca di recuperare il reale con l'ausilio o no della menzogna, ma sí di fissarlo in un momento: la fanciullezza.

Il critico Giuliano Manacorda nella sua Storia della Letteratura Italiana Contemporanea (1940-65) annovera Elsa Morante nel capitolo che ha come titolo: "Dalla delusione delle cose alla sublimazione delle parole", e come sottotitolo: "La celebrazione dell'immagine e della parola". Manacorda dà il suo giudizio sulla narrativa morantiana:

La vena poetica lucida e persino meticolosa nella descrizione della realtà, ma che muta poi quell' attenta precisione in uno sguardo penetrante e un po' allucinato che della realtà finisce per darci i segreti riposti, le corrispondenze impalpabili, le 
immagini apparentemente distorte ma nel profondo amaramente vere. $\mathbf{E}$ come un disperato e ripetuto tentativo di riafferrare il senso della vita al di là del superficiale fenomeno, con una conclusione generalmente spenta e crudele sulle cose e sugli uomini, inquieti protagonisti di eventi che essi non vogliono o non afferrano, illusi autori di una realtà che non si sa mai dove cominci ad essere fittizia ma che è in ogni caso avversa. ${ }^{13}$

Questo carattere della prosa si ritrova anche nei racconti de Lo scialle andaluso. Scritti fra il 1935 e il 1951, sono in realtà anteriori ai due romanzi già visti, ma furono pubblicati nel 1963.

Lo scialle andaluso "ì il racconto più lungo della raccolta dal titolo omonimo. Il tema è costituito dal rapporto madrefiglio che richiama vagamente il romanzo Agostino di Alberto Moravia. Tuttavia, se i sentimenti di Agostino sono palesemente erotizzati (l'identificazione della madre con la prostituta), quelli di Andrea sono privi di erotizzazione anche se altrettanto possessivi. Si potrebbe invece scorgere una certa affinità con Arturo: la mitizzazione della madre, la terribile delusione che genera il passaggio dall'infanzia all' adolescenza. Diversamente da Arturo però, l'adolescente An. drea non fugge di casa ma accetta con la madre il mondo.

Una giovane siciliana, Giuditta, vedova e madre di due fanciulli, uno dei quali è Andrea, è ballerina del Teatro dell' Opera di Roma. Geloso dell'amore materno per il teatro, Andrea si fa seminarista. Durante una passeggiata con i suoi compagni seminaristi Andrea vede su un manifesto pubblisizzante uno spettacolo di varietà la foto della madre. Spinto da un bollente desiderio di assistere ad una trionfale esibizione della genitrice, abbandona il seminario e va al teatro. Giuditta, ora attricetta matura e non più attraente, è fischiata dagli spettatori. Ella decide di abbandonare il teatro per dedicarsi esclusivamente ai figli. Euforico Andrea passerà tutta la notte avvolto nello scialle andaluso che la madre usava in ieatro.

Andrea, purtroppo, non tarda a comprendere che la rinuncia della madre al teatro non è stata per l'amore dei figli e sì perché nessuno le avrebbe offerto una scrittura. Cadono all'improvviso tutte le illusioni del ragazzo: "Andrea cominciò a capire che il suo patto con Giuditta, e tutta la sua vita precedente, gli avevano nascosto un inganno (p. 211).

13 MANACORDA, D. 330.

14 MORANTE. Elsa. Lo scialle andaluso. Torino. Einaudi, 1963. 520 p. Le cltazlonl sl referiscono auesta edizione e saranno cttati solo I numeri delle parine. 
"Per questi motivi, egli ebbe pietà di lei, e le perdonò. Ma il perdono che nasce dalla compassione è un parente povero del perdono che nasce dall'amore" (p. 212).

"Egli vorrebbe immaginare il futuro se stesso, e si compiace di prestare a questo Ignoto aspetti vittoriosi, abbaglianti, trionfi e disinvolute! $\mathrm{Ma}$, per quanto la scacci, ritrova sempre là, come una statua, un'immagine, sempre la stessa, importuna:

un triste, protervo Eroe avvolto in uno scialle andaluso" (p. 213).

Le vicende qui sono meno legate ad atmosfere fiabesche e fantastiche. Lo scialle andaluso, che racchiude le illusioni del teatro, è uno dei pochi elementi emblematici.

Scritto in terza persona, non si può affermare però che la tecnica narrativa sia completamente diversa da quella usata dall'io narrante. Si hanno, difatti, due piani narrativi: il piano di sviluppo azione e intreccio, e il piano di commento a quel'intreccio e a quell'azione.

La narrazione spesso tende all'identificazione della terza persona con il protagonista della narrazione; a volte l'intervento del narratore è palesemente in prima persona: "Mi duole, ma proprio dell'empietà di tale specie, né più né meno, fu capace di pensare là su quella sedia usurpata del Teatro Gloria, colui che aveva presunto non molto tempo prima d'essere sulla via della santità" (p. 270).

L'immedesimazione non è però a tal punto da imprimere al racconto una direzione monoprospettica; altrettanto frequentemente, la narrazione tende ad una registrazione delle cose e dei fatti, siano di esempio le trascrizioni fonografiche del parlato.

La narrazione comporta intersecazione, alternanza, a volte anche una coincidenza di due punti di vista: quello del protagonista e quello di chi narra oggettivamente:

$\mathrm{E}$ como ha potuto, senza vergogna, mentire, dicendo alla ragazza dal berrettino di avere appuntamento con Febea! Lui sapeva benissimo di mentire non solo nei riguardi del vero, ma anche nei riguardi del possibile! E chiaro come il giorno, ormai, che Febea (tanta è la sua noncuranza verso Andrea Campese), pur se pregata, avrebbe rifiutato di concedergli un appuntamento; e adesso, se chiamata a testimoniare dalla ragazza del berrettino, sarebbe pronta a smentire i vanti di lui; esarebbe fieramente annoiata di sapere che là in teatro, c'è quell'in- 
discreto, quel pretino travestito; e se qualcuno le annunciasse, nel suo camerino: "C'è qui fuori un certo Andrea, venuto a trovarvi", lei direbbe: "Chi? Andrea? Mai conosciuto. Ditegli che non ricevo, e fate che se ne vada" (p. 269).

Anche il trattamento del tempo presenta un bifrontismo: indicazioni temporali ora generiche, ora specifiche. Al tempo che fluisce nella imprecisione, si accavalla quello della puntualizzazione cronologica e, se il primo è dovuto all'indiferenziazione temporale di importantissimo valore segnaletico: una vita disingannata, il secondo costituisce un'accettazione all'illusione.

Il mondo salvato dai ragazzini (1968) vince il premio Zafferano. Contiene poesie, canzoni ed un atto unico. Importante è la nota introduttiva che riprende le tesi già espresse dalla Morante nella conferenza Pro e contro la bomba atomica. Il libro è una premessa indispensabile alla lettura della Storia, romanzo che uscirà nel 1974.

Il mondo salvato dai ragazzini è un libro-poema che rifiuta l'alienazione prodotta dalla civiltà atomica, smantella il grande carnevale della storia e sublima gli anticonformisti, umili, gli artisti.

Il nostro tempo, afferma la Morante, è espresso dalla bomba atomica, quasi che l'umanità si compiaccia della occulta tentazione di disintegrarsi, non per l'istinto del Nirvana predicato dalle filosofie orientali, raggiunto con la contemplazione e con la rinunzia ai valori mondani, ma con la disintegrazione della coscienza per mezzo della ingiustizia e demenza organizzate, dei miti degradanti, della noia convulsa e feroce..$^{1-}$ La storia è la violenza, l'annientamento della personalità, l'irrealtà è la poesia e i ragazzini. Anche il poeta infatti è un giovine di cuore.

La morte dei sensi è illusoria- una trasformazione nel ciclo perenne della vita. La morte-alienazione è la vera antagonista. Questa morte-alienazione costituisce il grande tema de: Il mondo salvato dai ragazzini. Le illusioni e le visioni de: L'isola di Arturo, così come la "menzogna" e il "sortilegio" del primo romanzo sono sostituiti dalla rivelazione impietosa del mondo, un mondo che è inferno e il cui cielo non è altro che una "bassa tenda cenciosa sopra il mondo". ${ }^{16}$

Le canzoni popolari che occupano più della metà della del libro scaturiscono da un anarchismo antistoricistico. Nella canzone "Il mondo salvato dai ragazzini", che dà il

15 MORANTE. Elsa. Pro o contra la bomba Btomica. Europa Letteraria, Roma, 30 mar. 1964

16 MORANTE, Elsa. Il mondo salvato dai ragazaini. Torino, Elnaudl, 1968. D. T9. 
titolo al libro, la storia è divenuta la "Grande Opera", un'immensa fantasmagoria teatrale, una mescolanza contraddittoria e senza senso di tragedia-farsa-parodia. Tutti sono attrat$t i$ al recitare e ne viene una confusione: litigi, aggressioni, persino i morti rimangono in piedi tanta è la violenza della moltitudine. L'unico a non lasciarsi attrarre dalla Grande Opera è Pazzariello, un ragazzo di borgata semideficiente che va in giro per le strade suonando sull'ocarita "Cielito lindo". La purezza, la demenza, la giovialità di Pazzariello sono la giovinezza aculturale che sfida la storia e la sua illusoria rappresentazione.

Le successive composizioni rivelano un crescendo di dolore. Nella "Sera Domenicale" Elsa Morante congeda la ragione perchè desidera passare la domenica con la follia. Anche l'atto unico de $I 1$ Mondo salvato dai ragazzini: "La serata a Colono" è sconvolgente. La scena è in una Colono che è l'astanteria di un manicomio-ospedalelager. Parallelamente alla tragedia greca, a Colono giunge Edipo, ricco proprietario meridionale, accompagnato da Antigone, sua figlia. A Edipo che rigetta la vita ed il suo simbolo, il Sole, accecandosi, si contrappone Antigone, la giovinetta semianalfabeta che ha della vita la concezione semplice o ingenua. Quasi alla fine dell'atto unico, a Edipo, mancato suicida, dopo aver bevuto una pozione datagli dalle suore dell'ospedale, appaiono le Benigne, le fate del luogo che gli mostrano una scala colorata con molte porte simbolizzando: i ritorni, il focolare, l'amore, la preghiera, la morte, il nulla. Edipo, gravato dal dolore del mondo, sceglie la porta del nulla "Io volevo/ tornare al corpo dove sono nato".'i Mentre Edipo soccombe e precipita nel nulla, nel vuoto, Antigone resiste al sopruso della storia.

Il libro ha senza dubbio un nesso con la produzione precedente della Morante, ma la presa di posizione anarchica si ciante schiettezza. Eppure trapela nel libro un barlume di verso: la propria Morante parla in prima persona con agghiacciante schiettezza. Eppure trapela nel libro un barlume di speranza contro l'avanzante alienazione, ce ne dà una prova la "pietas" di Antigone: un tentativo di sovvertire o, per lo meno, di uscire da una concezione tutta negativa del mondo.

La Storia," libro questo che è stato il "best-seller" in Italia e all'estero. Pubblicato nel 1974 dalla casa editrice Giulio Einaudi, Torino, qui in Brasile porta il titolo A História, traduzione di Wilma Freitas Ronal de Carvalho, diritti di pubblicazione esclusiva in lingua portoghese nel Brasile acqui-

17 MORANIE, II mondo salyato..., D. 96.

18 MORANTE; Elsa. La storia. Torino. Elnaudi, 1974. 665 p. Le citazionl sl rlferlscono a questa cdizione e saranno cltatl solo i numero delle pagine. 
stati dalla Distribuidora Record de Serviços de Imprensa S.A., Rio de Janeiro, con 604 pagine.

Il libro è stato al centro di una sproporzionata polemica. Bo, Del Buono, la Ginzgurg, Milano, Salinari, Soldati e molti altri, hanno affermato che si tratta di un libro destinato a durare. Di contro, Barilli, Balestrini, Pasolini, Siciliano, Spagnoletti ed alcuni altri hanno espresso più o meno apertamente il loro dissenso.

Riprendiamo La Storia prescindendo dalle polemiche che ne sono derivate, non senza però esporre il nostro punto di vista generale: è un libro che presenta grossi limiti e grossi pregi. I limiti sono in prima istanza di carattere ideologico ed anche politico, dato che Elsa Morante tende a sconfinare anche nel campo politico, nonostante le sia sostanzialmente estraneo ogni senso della dialettica storica. I limiti ideologici, tuttavia, spesso si traducono in punti di forza sul piano della realizzazione artistica.

La Storia si svolge nella Roma degli anni 1941-1947. Non è una Roma nella sua integrità e totalità di "Urbs" per antonomasia, non la Roma "eterna" che fa la storia, ma all'interno di quella stessa, la Roma che da millenni la subisce. E l'altra Roma, popolare e periferica che soffre l'orrore della fame, della violenza tedesca e fascista, della guerrigila partigiana.

Ida Raimundo è una maestra elementare. Vedova, a Roma si sforza per alimentare ed educare l'unico figlio che sta frequentando il liceo. La guerra è per lei la fame. Il tesseramento dei viveri costringe la povera gente a fare lunghe file davanti ai negozi e spesso a ricorrere alla borsa nera. Un'altra preoccupazione, però, assilla Ida: il pericolo di essere deportata in un campo di concentramento, visto che il suo sangue non è puro per essere di madre ebrea. Tale timore diventa ossessione soprattutto perché vive in assoluta solitudine. La situazione si fa ancor più dolorosa allorchè un soldato tedesco ubriaco, di passaggio per Roma, riesce a violentarla. Nasce un bambino che si chiamerà Useppe, o meglio, Giuseppe. Nel frattempo gli alleati cominciano a bombardare la città e Ida è costretta a rifugiarsi, con molti altri senza tetto, nello stanzone di una scuola, in una lontana borgata. Qui il bambino comincia a scoprire il mondo. Nino inizia la sua vita avventurosa. Arruolatosi volontario con $i$ fascisti, passerà poi ai partigiani. In ultimo si dedica al contrabbando. Gravemente ferito in un incidente automobilistico, durante uno scontro con la polizia, muore all'ospedale. Sempre allegro, pieno di fiducia in sé stesso, Nino aveva avuto due grandi affetti: il fratellino e la cagna Bella, che resterà l'amica e la protettrice del bambino. 
La povera maestra finalmente rientra in Roma. La deportazione degli ebrei romani aumenta la sua angustia che continua anche durante la notte accompagnata da incubi.

Giuseppe, o Useppe, muore nel quinto anno di vita per un attacco di epilessia. Tornando dalla scuola la madre lo incontra morto accanto a Bella. Dopo alcuni anni anche Ida morirà in un manicomio.

Il periodo di storia che la narrazione abbraccia è quello dell'ultimo conflitto, ma si tratta di una pura convenzione temporale: in verità "questo scandalo che dura da diecimila anni", abbraccia l'intera storia dell'uomo: il passato, il presente, il futuro. Non avviene alcun cambiamento e le vittime sono sempre le stesse.

Ogni personaggio rappresenta una storia propria ma anche generale, e la sua voce è la voce di tutti e si fa rappresentante di quella umanità esemplare alla quale Cèsar Vallejo dedica il verso ripreso da Elsa Morante ad apertura del libro - "Por el analfabeto a quien escribo".

Il romanzo si divide in nove lunghi capitoli, ognuno dei quali è datato ad un anno del conflitto ed è preceduto da un resoconto scheletrico degli eventi mondiali. Il libro si presenta, pertanto, come un componimento misto di storia e invenzione; si osserva però, immediatamente, che in quanto la storia delle vittime è narrata nei suoi particolari, la storia ufficiale è drasticamente relegata ai margini. Anche i riferimenti aile vicende pubbliche, innestati alla narrazione delle vicende private, obbediscono a criteri programmaticamente semplificatori. $\mathbf{E}$ evidente che Elsa Morante non si preoccupa di dare una interpretazione degli eventi secondo una logica oggettiva, $\mathrm{ma}$ in base a leggi proprie: ricostruire sei anni di storia, simbolici dei precedenti diecimila, col presupposto che l'uomo non è l'ar'tefice e sì la vittima della storia.

La storia è jl sostegno dell'invenzione ed è chiamata esplicitamente in causa nei sommari che aprono ogni capitolo. La storia si avvera attraverso atti crudeli come la guerra e l'odio. Tuttavia da questa rappresentazione di un dramma che si ripete e si ripeterà, si aprono pause di dolcezza: sono le pagine dedicate ai personaggi bambini. L'infanzia è per la scrittrice il momento della vita più vicino alla realtà, è l'innocenza veggente più atta a scoprire il senso delle cose. L'infanzia, ricca dell'originaria capacità dell'intuizione e non contaminata da convenzioni scopre di immediato il senso della realtà e lo interpreta in modo esatto.

Affidando la scoperta delle verità ai bambini, Elsa Morante istituisce un rapporto antitetico con gli adulti. NJel suo secondo romanzo L'isola di Arturo, l'innocenza fiduciosa del bambino protagonista muore man mano che il bambino di- 
venta adulto; ne Il mondo salvato dai ragazzini, in cui è rappresentato il fracasso della civilizzaione, la voce più potente è data ai bambini. Nel romanzo La Storia Carulì, la mammabambina ed Useppe, il figlio di Ida, sono al centro di questo universo infantile. In questo universo infantile convergono tutti i motivi della tragedia che scuote il mondo.

Nel romanzo sono segnalati i pensieri ed i sentimenti dei personaggi, così come le caratteristiche fisiche, e la trama degli avvenimenti esterni assorbe questo tessuto interiore che entra a far parte della storia tanto quanto gli eventi decisivi. La trama è semplice, si direbbe che è la storia del piccolo Useppe e della cagna Bella. Di fronte al bambino la cagna ne è quasi il complemento. Anche se il romanzo ci parla continuamente della maestra elementare, paragonata con Useppe, Ida diventa un personaggio secondario, nonostante sia di grande aiuto per la comprensione del messaggio. E chiaro, però, che la storia è la vera protagonista!

Lo studio del personaggio Ida ci rivela una passività allucinata e angosciosa. Donna senza ideologie, tutta risolta nella essenziale biologia di madre "Ida era rimasta, nel fondo, una bambina, perché la sua precipua relazione col mondo era stata e rimaneva, consapevole o no) una soggezione spaurita. I soli a non farle paura, in realtà, erano stati suo padre, suo marito, e più tardi, forse i suoi scolaretti" (p. 21).

Gli effetti di questo timore non si notano tanto negli atti di Ida, quanto nei suoi sogni. Questa lettura al rovescio del personaggio ha una funzione oggettivamente critica nei confronti di una società violenta, disumana. Vediamo, per esempio, uno dei sogni di Ida:

Non appena si addormentava, come al crollo di una parete divisoria, subito ricominciava il suo notturno viaggio labirintico, senza né vuoti né riposi. Eccola che capitava in un terreno vago, una sorta di periferia, con qualche figura provvisoria di costruzioni. Lei è la sola vestita in mezzo a una folla di gente nuda, tutta in piedi, coi corpi ammassati uno all'altro senza spazio di respiro. E lei si vergogna d'esser vestita, per quanto nessuno sembra accorgersi di lei. Tutta quella gente pare abbacinata, coi volti gessosi e fissi in un'assenza degli sguardi e delle voci, come se ogni mezzo di comunicare con loro fosse scaduto. Lei piange, così che il suo singhiozzo altissimo è l'unico presente; ma appunto perché è il solo, pare che rida...

Ecco però che la risata non viene più da lei, che sta sola dritta, come una marionetta, fra certi 
mucchi di travi e di pietrisco. Non si vede nessuno, ma sotto quei mucchi si sente un fragore come di migliaia di denti che masticano, e sotto a questi il lamento di una creatura che lei non può aiutare, per quanto ci si sforzi, perché i suoi ginocchi sono rigidi come fossero fatti di legno. Finalmente, la risata si confonde nell'abbaiare di un cane, forse è Blitz, che fruga disperato per liberare Ninnarieddu e Giuseppe. Ma a questo punto lei si trova caduta in un locale sotterraneo, dove rimbomba una musica assordante, orribilmente comica, che la costringe a ballare. $\mathrm{E}$ nel ballo essa deve mostrare le gambe, ma tenta di coprirsele, sapendo di avere certe cicatrici infami che la deturpano e per le quali verrà punita fino alla settima generazione" (p. 128).

Ida reinventa la realtà attraverso la sua mente immatura e deformata. $\mathrm{Ci}$ sono anche momenti in cui Ida vive attimi di fuga da se stessa e dalla schiavitù storica a contatto con il valore epifanico delle cose:

Lei s'era staccata dal continente affollato e vociferante della sua memoria, su una barca che in questo intervallo aveva fatto il giro del mondo; e adesso, risalendo allo stesso scalo della partenza, lo ritrovava silenzioso e quieto. Non c'erano più urla di folla, nessun linciaggio. Gli oggetti familiari, spogliati da ogni affetto, non erano più strumenti, ma creature vegetali o acquatiche, alghe, coralli, stelle marine, che respiravano nel riposo del mare, senza appartenere a nessuno (pág. 71).

Osserviamo adesso la reazione di Ida chiamata a riconoscere il figlio Nino all'obitorio: "All'atto di riconoscerlo, la sensazione immediata. . fu una feroce lacerazione della vagina, come se di nuovo glielo strappassero di là. Diversamente da quello di Useppe, il parto di Nino era stato terribile per lei" (p. 464).

Ecco dopo la scena dell'obitorio, la seconda sensazione semicosciente che le resterà di quella mattina:

Lei non poteva urlare, era diventata muta, e transitava per certe strade irriconoscibili, dove la luce era uno zenith accecante, che dava a tutti gli oggetti un rilievo osceno. Le foto esposte nelle edicole ridevano oscenamente, la folla si contorceva e le statue numerose in cima alla basilica si avventavano 
verso il basso in atteggiamenti mostruosi... Quelle statue erano le stesse che aveva vedute nei giorni che era nato Useppe, però oggi la basilica s'era fatta storta e cosi pure le altre case e costruzioni all'intorno, come da specchi convessi (p. 464).

Nulla di tutto ciò accade invece a Úseppe, il personaggio cui poeticamente confluisce tutta la vicenda di Ida non meno di quelle dei personaggi minori. C'è in lui come una silenziosa e pensosa attitudine e ci si commuove dinanzi alla sua anima aperta, alla sua sorpresa per l'immenso spettacolo del mondo. Quello che per Nino è ansia di vivere, diventa in Useppe necessità di scoprire ed ogni scoperta è meravigliosa. Malaticcio sin dalla nascita, ancor più indebolito per il sopravvenire dell'epilessia che in poco tempo lo annienterà. Commovente la lotta che per lui si combatte ogni giorno dalla madre per nutrirlo e dall'altra "madre", la cagna Bella per proteggerlo.

Un personaggio che ha un ruolo di contrasto, paragonato con gli altri è Nino, il figlio maggiore e legittimo di Ida. Nino non sembra essere segnato dal destino delle vittime. L'esuberanza della vita lo spinge prima ad allistarsi, volontario, nelle file fasciste, più tardi, invece, diventerà partigiano comunista "Noi siamo della generazione della violenza. Noi siamo la prima generazione dell'inizio!" (p. 57). In seguito comprende che non si può credere in nulla e che tutti i caporioni, come li chiama, di destra e di sinistra, si equivalgono. Eppure il cinismo di Nino scompare dinanzi all' amore estatico di Useppe che rappresenta per lui l'unica cosa pulita.

Diversamente dagli "incoscienti" Nino e Useppe, Davide Segre, un ebreo la cui famiglia è stata massacrata in Germania in un campo di sterminio, rappresenta la consapevolezza. Qui è la scrittrice che parla per interposta persona. Intellettuale anarchico, Davide si ritiene capace di giudicare il presente ed il passato. Il personaggio è ben riuscito, specie nell'autocritica disperata, nonostante espressa in un modo piuttosto confuso, e nella proposta utopica " $\mathrm{E}$ dunque che ci vorrebbe, per la Rivoluzione totale? niente, un movimento elementare di due secondi, come ridere o stirarsi appena svegli! basterebbe riconoscere il Cristo in tutti quanti: io, te, gli altri..." (p. 592).

Anche Davide sarà vinto: la droga lo ucciderà.

$\mathrm{Ma}$ ecco che Elsa Morante, nello scandalo della Storia, ci insegna a riconoscere quei sentimenti di bontà che stanno al di là di ogni ideologia e ci ricordano la verità e la bontà della natura. Verità e bontà che si identificano nella gatta 
del capannone dei rifugiati, o nella cagna Bella che s'investe del ruolo di madre. $E$ più ancora, la vecchia ebrea che supplica di essere caricata anch'essa nel vagone piombato, ed il soldato Giovannino che muore nelle steppe gelate e nel delirio vede sua madre uscire dalla casa con la rocca sotto il braccio ed in mano il fuso; e soprattutto Useppe che muore prima che venga a rompersi il suo perfetto accordo col mondo della natura.

In questo romanzo la pietà clella scrittrice non è una "pietas" consolatrice perché essa si origina nella coscienza dell'assurdo: i suoi personaggi camminano nella storia inconsapevoli verso una meta che è la loro cancellazione dalla vita.

Aracoeli ${ }^{19}$ è l'opera recente della Morante, finita di essere stampata il 23 ottobre 1982 per conto della Giulio Einaudi editore.

Circa_otto anni sono passati dalla Storia, otto anni di ferviao silenzio tre dei quali hanno tenuto la Morante nella tormentata immobilità di un letto.

L'opera ha subito l'esplosivo della polemica: c'è chi vede nel libro un evento memorabile nella narrativa italiana di questi tempi, c'è chi lo accusa di "moravieggiare" ostinatamente, c'è chi esige che il libro venga accostato con rispettosa cautela e, se possibile, con qualche amicizia perché, prima ancora di essere persuasi, dalla lettura ci si esce turbati.

Aracoeli è la storia del protagonista-narrante il quale, sui quarant'anni, va in cerca delle memorie della madre perduta: la spagnola Aracoeli.

Nella nostra modesta opinione, l'opera non è certamente superiore a La Storia, né ha lo stupendo protagonista de L'isola di Arturo, è però un lavoro đi intima, dolorosa natura che la propria Elsa Morante vorrebbe, a torto, che fosse letto come un libro postumo.

Manuele, nell'illusoria speranza di trovare fuori dalla propria nevrosi un'immagine di salvezza "Rannicchiarmi dentro di lei, nell'unica mia tana, persa ormai chi sa dove" (p. 18). parte alla ricerca della madre Aracoeli che pure sa morta.

Sulle origini familiari di mia madre, e sulla sua esistenza prenuziale di vergine paesana, vigeva difatti, in casa nostra, una sorta di onorabile segreto di stato, di cui mio padre era stato l'unico depositario legittimo e la zia Monda nient'altro che una semplice fiduciaria, con uffici reservatissimi e limi-

19 MOrante, Elsa. Aracoell. Torino, Einsudl, 1982. 328 p, Le citazioni si riferiscono a questa edizlone c saranno citatl solo 1 numeri delle paginc. 
ti di stretta necessità. Si trattava invero di un segreto obbligato e per nulla tenebroso, in se stesso; ma la fantasia infantile non può figurarsi un segreto se non aumentato di tenebre o circonfuso di splendori; i quali rischiano di scadere non appena l'arcano affronti la luce. La sua storia mi era stata trasmessa, fino da quando io le crescevo nell'utero, attraverso lo stesso messaggio cifrato che aveva trasmesso dalla sua pelle alla mia il colore moreno. $E$ sarebbe stato vano, dunque, tentare una traduzione terrestre di quanto io portavo, congenito, dentro di me, già stampato nel proprio codice favoloso (p. 23).

Con Manuele sofferente, sotterranea figura, fallito funzionario di casa editrice, omosessuale infelice, nell'avvelenata bivalenza del suo edipico amore (ha saputo anche odiare la madre):

E finalmente vedo adesso tutto la ridicolaggine di certi miei contorcimenti assurdi, nei tentativi ripetuti di uscire dalla mia pelle.E delle tante camminate e rincorse e accattonaggi di me che andavo mendicando risposte di amore, contro ogni riconferma spietata della Necessità. Ele attese incalcolabili di una smentita. $\mathrm{E}$ le ricadute. $\mathrm{E}$ i sorrisi confidenti davanti a facce fredde. $E$ le povere gratitudini per concessioni svogliate. $\mathbf{E}$ i brividi alle nuove ripulse. E le presunzioni irrisorie. Niente. Nessuna risposta. Da quando ho perso il mio primo amore Aracoeli, mai più mi si è dato un bacio d'amore (p. 47).

E più avanti Manuele dirá: "La mia prima disperata domanda fu sempre, infatti, di essere amato. $E$ se per un caso impossibile, mi si fosse dato una scelta, avrei voluto fra i due, piuttosto che il compratore essere la merce" (p. 63).

Il romanzo si riduce ad essere un monologo sregolato. Ad un tempo attuale, il 1975, s'intersecano i piani del passato e i ricordi del vissuto. Questo ricordo del vissuto è frantumato, sconvolto, deriso.

Il sistema androgino della Morante è vinto. Se nei romanzi precedenti esso non ammetteva gli uomini, in quest'ultimo non ammette più nulla. Manuele afferma che a ripensarci, pure da adulto, non ne ha mai saputo molto di piú che a sei 0 a dodici anni "Io sono un animale schiacciato sulla schiena da una grossa pietra. Con le zampe disperate raspo 
la terra, e scorgo al di sopra, mezzo cieco, degli azzurri vapori. Non so perché sono incollato alla terra. Non so quale sostanza siano quei vapori. Non so chi mi ha scaricato addosso la pietra. Non so che animale sono" (p. 143). E in un crescendo doloroso, continuerà: "Alcune sequenze e figure oggi mi si proiettano davanti con la forza delle allucinazioni, precisandosi fino in certe minuzie, ammicchi, smorfie. Per tanti anni si finsero cancellate; e adesso vengono a esibirsi, quali documenti d'archivio, nel mio teatro vuoto. Che siano anche queste, finzioni?" (p. 242).

In ogni pagina del romanzo appare il nome di Aracoeli, persino più volte ripetuto in una stessa pagina. Il nome del protagonista-narrante, invece, è solamente conosciuto a pagina centotrentatrè, ossia quasi alla metà del romanzo. Sin dall'inizio sappiamo che il genitore di Manuele si chiama Eugenio Ottone Amedeo; di grande connotazione è il fatto che questi, poco menzionato, è spesso chiamato "Il Comandante" (è difatti un militare), rarissime volte: "mio padre", mai "papà". "Io invece non sono mai stato figlio di un padre. Evitavo sempre, con lui, di chiamarlo papà; nel suono stesso di queste due sillabe, mi si faceva sentire un che di ridicolo, quasi d'indecoroso. Le due sillabe ma-ma, invece, mi suonavano dolcissime e naturali, come voci proprie della mia carne" (p. 184).

Spagnola, sposata a un militare italiano che finirà alcolizzato, immagine di purezza, Aracoeli, dopo la morte di una figlia appena nata, è stata travolta da una cieca febbre erotica ed ha finito per prostituirsi. Il bambino Manuele, nonostante l'abbandono della madre, continuerà a vederla nei suoi sogni e nelle sue visioni, e questo edipico amore che frequentemente si rivolta in una nostalgia negativa, di rigetto, accompagnerà tutta l'esistenza di Manuele: "Dopo tanti anni, io (sempre uguale) resto ancora bersaglio scoperto del suo supplizio irrisorio" (p. 149). Manuele andrà a cercarla non nel cimitero di Roma, il Campo Verano, devastato da un bombardamento aereo, ma al suo luogo di nascita: El Almendral, un paesetto sperduto che non si trova in nessuna carta: "Io mi domando perfino se con questo viaggio, sotto il folle pretesto di ritrovare Aracoeli, io non voglia piuttosto tentare un'ultima, sballata terapia per guarire di lei. Frugare nelle sue radici finché s'inaridiscano sotto le mie mani, poiché di estirparle non sono capace" (p. 149).

Sotto le apparenze di un viaggio immaginario ed insieme reale di un figlio alla ricerca della madre, Aracoeli è un romanzo di castrazione.

Cosi come nei precedenti romanzi, anche qui vi è il rapporto madre/figlio, ma l'abbraccio Nunziata/Arturo de L'Iso- 
la di Arturo; Ida, Useppe, de La Storia; si scioglie qui Aracoeli Manuele, in una straziante lacerazione. Dice il protagonista che a volte, specie in certe solitudini estreme, nei vivi prende a battere una pulsione disperata, che li stimola a cercare i loro morti non solo nel tempo, ma anche nello spazio. C'è chi li insegue all'indietro nel passato e chi si protende al miraggio di raggiungerli in un futuro ultimo; e c'è chi, non sapendo più dove andare senza di loro, corre i luoghi, su una qualche loro pista possibile:

Sono tentato a inseguire la mia ragazza Aracoeli in tutte le direzioni dello spazio e del tempo, fuorché una a cui non credo: il futuro. In realtà, nella direzione del mio futuro, io non vedo altro che un binario storto, lungo il quale il solito me stesso, sempre solo e sempre piu vecchio, seguita a portarsi su e giù, come un pendolare ubriaco. Fino a quando sopravviene un urto enorme, ogni traffico cessa. $E$ il punto estremo del futuro. Una sorta di mezzogiorno accecante, o di mezzanotte cieca, dove non c'è più nessuno, e nemmeno io (p. 24).

Anche se la storia è a volte farraginosa, siamo attratti dalla scrittura stupenda, dalla malia della sua prosa tra i cui frammenti fermenta tutto il mistero senza fondo della vita.

Così come il nome "Aracoeli", i vocaboli "specchio" e "occhiali" sono altamente significativi, anzi, sono le parolechiavi. Dice Manuele che secondo certe negromanti, gli specchi sarebbero delle voragini senza fondo, che inghiottono, per non consumarle mai, le luci del passato. Sull'aereo per Almeria, Manuele impara che "almeria" in arabo significa specchio. Ciò gli appare un nuovo segno del destino, chiaro simbolo della specchiera da cui sempre gli riaffiora, viva e presente Aracoeli. Un istinto di rancore vendicativo lo ha forzato davanti allo specchio, a riguardarsi da vicino, esposto nella sua nudità e guardandosi negli occhi non ha scorto altro che "la piccola ombra diluita (quasi naufraga) di quel solito "ninho" tardivo che vegeta segregato dentro di me. Sempre il medesimo, con la sua domanda d'amore scaduta e inservibile, ma ostinata fino all'indecenza (p. 107).

A guisa dello specchio, anche gli occhiali fanno parte della simbologia. Per difendersi da intrusioni esterne Manuele si toglie gli occhiali "Ho riposto gli occhiali nella tasca della giacca, così che gli altri passeggeri della corriera mi si riducano a larve informi" (p. 52). Eppure, con e senza gli occhiali, il protagonista vede sempre attorno una valanga di rifiu- 
ti "Io mi metto, e mi ritolgo e mi rimetto gli occhiali, per ritrovare sempre il solito sfacelo di sassi e rupi" (p. 131).

Allorché il "balio" Daniele, incuriosito degli occhiali di Manuele, se li prova, esclama, togliendoseli in fretta: "Ci si vede tutto coperto e confuso", Manuele commenta poi tra sé "Era così che io vedevo il mondo, invece, senza gli occhiali" (p. 223).

Anche lo "chichón", forte liquore spagnolo, la bevanda più volte bevuta da Manuele, racchiude una forte connotazione, rappresenta il filtro per un sortilegio: la magica svolta al passato, il rientro nel corpo della madre.

Per alcuni lettori il romanzo è un libro ossessionato dal sesso, diremmo piuttosto che il sesso non vi è esaltato ma sì flagellato.

Il senso del romanzo Aracoeli ci viene rivelato dall'intuizione del protagonista "In verità, di tutte le voragini fra cui ci moviamo alla cieca, nessuna è tanto cupa, e per noi stessi inconoscibile, quanto il nostro proprio corpo. Lo si definì un sepolcro, che ci portiamo appresso; ma la tenebra del nostro corpo è più astrusa per noi delle tombe" (p. 320).

Elsa Morante appartiene a quella categoria di scrittori che non amano parlare di sé, dei propri libri. Gelosa della propria vita privata, è convinta che i libri non si possono né si devono spiegare: o parlano da sé, comunicando qualcosa a chi li legge, o sono nient'altro che cadaveri di cui non vale la pena di occuparsi. Dice: "Vorrei essere soltanto una voce che corre per il mondo. Mi piacerebbe che i miei libri andassero in giro anonimi, o che magari li raccontasse un cantastorie" 20

L'otto aprile del corrente anno, Elsa Morante ha tentato il suicidio nella sua casa romana di via dell'Orca. A pochi mesi dalla pubblicazione di Aracoeli, una risposta al gesto dell'autrice può essere forse incontrata a pagina centocinque del romanzo citato, allorché Elsa fa domandare al suo personaggio se il risultato di ogni analisi dei nostri "moti" manifesti non dia risultati immaginari: "Pare facile conoscere perché si piange. $\mathrm{Ma}$ in realtà, chi volesse, potendo, esaminare il "seme del pianto", si perderebbe-io temo-in un'analisi oscura e confusa, negata a ogni formula chimica. Peggio, ancora, poi, se quel povero seme è ormai secco".

La cronaca ha le sue esigenze, specialmente quaundo si tratta di un nome come quello della Morante, eppure, al di là dell'evento di cronaca, noi che siamo suoi lettori, collocheremo la risposta a questo "moto manifesto" in un clima poetico...

20 GRieco, G. Parla Elsa Morante, la scrittrice misteriosa. Gente, Torino, 12 nov. 1982. p. 25-7. 


\section{RESUMO}

O presente estudo abrange toda a obra de Elsa Moran te, incluindo o romance Aracoeli, publicado há poucos meses.

Salientam-se os núcleos caracterizantes da obra moran. tiana: a colocaçāo do homem em uma dimensāo condicio. nante, a sensação do mistério da vida e do nosso inútil questioná-la, o consequiente desnortelo do homem e a expansão para o fabuloso. Nas primeiras obras a narrativa se produz no desequilibrio de estupefato lirismo consonante com uma atmosfera surrealista, todavia, essa reinvenção da realidade origina-se de uma licida leitura "pelo avesso" da realidade cujas imagens sāo intencionalmente torcidas.

A partir de $\mathbf{O}$ mundo salvo pelas crianças, as ilusões e as visóes de $A$ ilha de Artur, assim como a "mentira" e o "sortilégio" do romance homônimo, são substituídos pela revelação impiedosa do mundo. Desmantela-se o "grande Carnaval" da história. Contudo, se no penúltimo romance: A História, os anticonformistas e os humildes, quase destroços de um naufrágio, ensinam-nos a reconhecer a verdade e a bondade da natureza, na recente produção: Aracoeli não se admite mais nada. $O$ romance torna-se um monólogo descomedido.

\section{RIFERENZE BIBLIOGRAFICHE}

1 FALQUI, Enrico. Novecento letterario. Firenze, Vallecchi, $1961.630 \mathrm{p}$

2 GRIECO, Giuseppe. Parla Elsa Morante, la scrittrice misteriosa. Gente, Torino, 12 nov. 1982.

3 LUKACS, György. Il significato attuale del realismo critico. Torino, Einaudi, 1957. $425 \mathrm{p}$.

MANACORDA, Giuliano. Storia della letteratura italiana contemporanea. Roma, Ed. Riuniti, 1972. 560 p.

5 MORANTE, Elsa. Aracoell. Torino, Einaudi, 1982. 328 p.

6 . Il gioco segreto. Milano, Garzanti, 1941. $422 \mathrm{p}$.

7 L'isola di Arturo. Torino, Einaudi, 1957. 427 p.

8 - Menzogna e sortilegio. Torino, Einaudi, 1948. 621 p.

9 . Il mondo salvato dai ragazzini. Torino, Einaudi, 1968. $560 \mathrm{p}$.

10 . Pro o contra la bomba atomica. Europa Letteraria, Roma, 30 mar. 1964.

11 . Risposta a dieci voci per il silenzio. Europa Letteraria, Roma, 30 gen. 1964.

12 Lo scialle andaluso. Torino, Einaudi, 1963. $520 \mathrm{p}$.

14 . Le straordinarie avventure di Caterina. Torino, Einaudi, 1941. $120 \mathrm{p}$.

15 PUPINO, Angelo. I contemporanei. Milano, Marzorati, 1973. 1227 p.

16 SABA, Umberto. Il Canzoniere. Torino, Einaudi, 1961. 321 p. 